\title{
Comparación de la concentración total de proteínas salivales de adultos y adultos mayores
}

\author{
Comparison of total salivary protein concentration in adults and older adults
}

\author{
Castro RJ ${ }^{1}$, Guzmán $\mathrm{G}^{2}$, Giacaman RA ${ }^{3}$
}

\begin{abstract}
RESUMEN
Objetivo: Entre las moléculas orgánicas que componen la saliva se han descrito un gran número de proteínas. No existe evidencia suficiente que permita especular sobre los cambios en el contenido proteico salival asociados al envejecimiento normal. El objetivo fue determinar si existen diferencias en la concentración total de proteínas en saliva de adultos y adultos mayores. Método: Se obtuvieron muestras de saliva estimulada y no estimulada de individuos pertenecientes a dos grupos etarios, adultos $(A)(n=30)$ (edad promedio: 25 años) y adultos mayores (AM) ( $n=30)$ (edad promedio: 68 años). Se excluyeron aquellos sujetos que presentaron alguna patología oral/sistémica o consumo de fármacos/sustancias relacionados con alteraciones del flujo o composición salival. Cada muestra fue centrifugada. A $10 \mu \mathrm{l}$ del sobrenadante se agregaron $190 \mu \mathrm{l}$ de solución de Bradford. Se realizó el recuento mediante lectura con espectrofotómetro $(595 \mathrm{~nm})$. La concentración de proteínas fue comparada entre los grupos en estudio utilizando la prueba $t$ de Student $(p<0.05)$. Resultados: La concentración de proteínas totales de saliva no estimulada fue mayor en AM (5.5 $\pm 2.6 \mu g /$ $\mu \mathrm{l})$ que en $\mathrm{A}(3.2 \pm 1.7 \mu \mathrm{g} / \mu \mathrm{l})(\mathrm{p}<0.05)$. La concentración de proteínas totales de saliva estimulada fue mayor en $\mathrm{AM}(4.9 \pm 0.4 \mu \mathrm{g} / \mu \mathrm{l})$ que en $\mathrm{A}(4.0 \pm 0.3$ $\mathrm{ug} / \mu \mathrm{l})(\mathrm{p}<0.05)$. Conclusión: La concentración de proteínas en saliva aumenta en adultos mayores. Se requieren nuevos estudios para caracterizar estos cambios salivales con la edad.
\end{abstract}

Rev. Clin. Periodoncia Implantol. Rehabil. Oral Vol. 5(1); 25-28, 2012.

Palabras clave: Proteínas salivales, saliva, envejecimiento, adultos mayores.

\begin{abstract}
Objective: A high number of proteins has been described in saliva. Scarce evidence allows to speculate about the variations on protein content associated with aging. The aim was to determine if there is a difference in total protein concentration between adults and older adults. Methods: Samples were collected from unstimulated and stimulated saliva of individuals from two age groups, adults $(A)(n=30)$ (mean age 25 years) and older adults $(O A)$ ( $n=30)$ (mean age: 68 years). Subjects with oral/systemic diseases or consuming medications that alter salivary flow were excluded from the study. Each sample was centrifuged and $10 \mu \mathrm{l}$ of the supernatant was added to $190 \mu \mathrm{l}$ of Bradford solution. Protein concentration was obtained by spectrophotometry (595 $\mathrm{nm}$ ) Protein concentration was compared between the groups using student's t test $(p<0.05)$. Results: Total protein concentration from unstimulated saliva was higher in $\mathrm{OA}(5.5 \pm 2.6 \mathrm{ug} / \mathrm{ul})$ than in $\mathrm{A}(3.2 \pm 1.7 \mathrm{ug} / \mathrm{ul})(\mathrm{p}<0.05)$. Total protein concentration of stimulated saliva was higher in $\mathrm{OA}(4.9 \pm 0.4 \mathrm{ug} / \mathrm{ul})$ than in $A(4.0 \pm 0.3 \mathrm{ug} / \mathrm{ul})(\mathrm{p}<0.05)$. Conclusion: Salivary protein concentration increases in older adults. Further studies are suggested to characterize these changes.
\end{abstract}

Rev. Clin. Periodoncia Implantol. Rehabil. Oral Vol. 5(1); 25-28, 2012.

Key words: Salivary proteins, saliva, aging, older adults.

\section{INTRODUCCIÓN}

La saliva juega un significativo rol en la preservación y mantención de la salud oral(1). Se compone de un $99 \%$ de agua y sólo un $1 \%$ de moléculas orgánicas e inorgánicas. Entre las moléculas orgánicas, se han descrito un alto porcentaje de proteínas ${ }^{(2,3)}$. Si bien, a la fecha se han descrito más de 1000 tipos diferentes de proteínas, sus propiedades bioquímicas para muchas de ellas, están aún en estudio(4,5), como así también la asociación de estas con enfermedades como diabetes ${ }^{(6)}$, fibrosis quística ${ }^{(7)}$, cáncer oral(8) y caries dental(9). Junto a lo anterior, se describe que el proceso de envejecimiento podría alterar el contenido proteico de la saliva ${ }^{(1,10)}$

Las proteínas salivales juegan un rol mayor en la dinámica de la cavidad bucal, ya que se les atribuyen propiedades antimicrobianas y antifúngicas, participan en la lubricación y mantención de la integridad de la mucosa, contribuyen a aumentar la capacidad buffer y promueven la remineralización, además de participar en los procesos fisiológicos de deglución, digestión, fonación y gusto(1). La importancia de analizar las variaciones existentes en la composición proteica salival, radica en que estas moléculas juegan un rol preponderante en la mantención de la salud e integridad tanto de los tejidos dentarios, como de la mucosa oral ${ }^{(11)}$.

Hasta ahora, poco se sabe de los cambios en el contenido proteico salival asociados al envejecimiento normal y no existe evidencia sustancial que permita especular sobre las variaciones esperables según el grupo etario. Un estudio, demostró que las características de las proteínas salivales en niños eran significativamente diferentes a las de los adultos, presentándose menor concentración de proteínas y número de bandas en geles de electroforesis a medida que avanza la edad de los sujetos ${ }^{(12)}$. Por el contrario, otra investigación reportó diferencias en el contenido y tipo de proteínas de la saliva de mujeres de distintas edades, presentándose mayores valores a mayor edad ${ }^{(10)}$.

El vertiginoso aumento de la población mayor en el mundo hace de este un tema de alta relevancia. En la actualidad, a nivel mundial los adultos mayores (personas sobre 60 años) representan el segmento poblacional con mayor proyección de crecimiento; esperándose que para el año 2050, la cantidad de personas mayores supere a la cantidad de personas jóvenes ${ }^{(13)}$. En Chile, el año 2010 los adultos mayores representaban el $13 \%$ de la población y se espera que para el año

1. Cirujano Dentista, MSc. Unidad de Cariología, Grupo de Investigación en Odonto-Geriatría GIOG. Departamento de Rehabilitación BucoMáxilofacial, Universidad de Talca. Talca, Chile.

2. Cirujano Dentista. Unidad de Cariología, Grupo de Investigación en Odonto-Geriatría GIOG. Departamento de Rehabilitación Buco-Máxilofacial, Universidad de Talca. Talca, Chile.

3. Cirujano Dentista, PhD. Unidad de Cariología, Grupo de Investigación en Odonto-Geriatría GIOG. Departamento de Rehabilitación BucoMáxilofacial, Universidad de Talca. Talca, Chile.

Correspondencia autor: Ramiro J. Castro Barahona. rcastro@utalca.cl. Escuela de Odontología, Universidad de Talca. 2 norte 685, Talca, Chile. Trabajo recibido el 28/12/2011. Aprobado para su publicación el 05/03/2012. Financiamiento: esta investigación fue financiada con recursos del proyecto número res.385 año 2011 de la Dirección de Investigación de la Universidad de Talca a Ramiro J. Castro Barahona. 
2050 este grupo crezca hasta un $28 \%^{(14)}$, presentando el índice de envejecimiento más alto de América Latina ${ }^{(15)}$. Para el 2050 se espera que la proporción de personas mayores llegue a cerca del $28 \%{ }^{(16)}$.

En la presente investigación planteamos un método sencillo para la determinación de la concentración de proteínas en la saliva, lo que pretende ser un primer paso para la exploración de diferencias cuantitativas y cualitativas en las proteínas salivales por grupo etario o por patología específica. Los hallazgos de este y otros estudios en curso servirán como base para el desarrollo de test diagnósticos o de determinación de riesgo de patologías de alta prevalencia como caries o enfermedad periodontal. Así, dada la falta de información disponible y considerando la potencial importancia diagnóstica de las proteínas salivales en diferentes grupos etarios, el objetivo de este trabajo fue determinar si existen diferencias en la concentración total de proteínas en saliva de adultos y adultos mayores.

\section{PACIENTES Y MÉTODOS}

Para realizar el estudio, se seleccionó una muestra por conveniencia de 60 individuos (30 de género masculino y 30 femenino) entre los pacientes que estaban en control médico-odontológico regular en los consultorios de la ciudad de Talca, Chile. Para ingresar al estudio, los sujetos debían leer y firmar un consentimiento informado. El protocolo del estudio y el consentimiento informado fueron aprobados por el Comité de Bioética de la Universidad de Talca.

Se conformaron dos grupos: Adultos (grupo $A ; n=30$ ), compuesto por 15 hombres y 15 mujeres, cuyas edades fluctuaron entre 18 y 39 años (promedio: $25 \pm 3$ años) y Adultos Mayores (grupo AM; $n=30$ ), compuesto por 15 hombres y 15 mujeres, cuyas edades fluctuaron entre 61 y 87 años (promedio: $68 \pm 6$ años).

A cada participante, se le realizó una anamnesis general, examen clínico extra e intraoral y los datos fueron registrados en una Ficha Clínica confeccionada para el estudio.

Se excluyeron del estudio a aquellos sujetos que presentaron alguna patología oral o sistémica relacionada con alteraciones del flujo o composición salival(17), consumo de fármacos de cualquier tipo que alteren la secreción salival(17), consumidores de alcohol(18), fumadores ${ }^{(19)} \mathrm{O}$ tratamiento de radioterapia y/o quimioterapia de cabeza y cuello(20).

\section{Recolección de Muestras Salivales}

A cada participante se le solicitó una muestra de flujo salival no estimulado y estimulado según el Protocolo del Departamento de Cariología de la Universidad de Malmo, Suecia. Para tal acción se les solicitó a los participantes que acudieran a la toma de muestra sin haberse lavado los dientes ni comido, al menos dos horas antes ${ }^{(19,21)}$. De ser necesario, sólo podían beber agua.

Todas las muestras de flujo salival fueron recolectadas en una sola sesión para cada individuo (con una diferencia de 20 minutos como mínimo entre estimulado y no estimulado), bajo las mismas condiciones y por el mismo operador entre las nueve y once de la mañana, con el propósito de reducir la influencia de los ritmos circadianos en el flujo

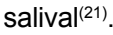

\section{Recuento de Proteínas}

Cada muestra fue recolectada en tubos milimetrados estériles de $15 \mathrm{ml}$ (Kima, Italia), rotulados con la edad y género del sujeto y mantenidas a $-20^{\circ} \mathrm{C}$ en un recipiente con hielo seco durante su recolección y traslado al laboratorio(22). Las muestras fueron centrifugadas (12.000 rpm por 20 minutos a $4^{\circ} \mathrm{C}$ ) y $10 \mu \mathrm{l}$ del sobrenadante depositados en una microplaca de 96 pocillos (Becton Dickinson, USA). Se efectuó la determinación del contenido de proteínas mediante un kit Bradford Reagent B6916 - for $1-1.400 \mu \mathrm{g} / \mathrm{ml}$ protein (Sigma-Aldrich, USA), siguiendo las indicaciones del fabricante. Brevemente: se agregaron $190 \mu \mathrm{l}$ de solución de Bradford a los $10 \mu$ de muestra y se incubó 30 minutos a temperatura ambiente. Una vez completado el tiempo, se efectuó la lectura con un espectrofotómetro (VICTOR ${ }^{\text {TM }}$ X - Multilabel Plate Reader, PerkinElmer, USA) a una longitud de onda de $595 \mathrm{~nm}$ y se proceso la información con el software Gen5 Data Analysis Software (Biotek, USA).

\section{Análisis Estadístico}

La normalidad en la distribución de los datos fue verificada con el test Kolmogorov-Smirnov el que indicó normalidad. Para comparar las diferencias entre los grupos de estudio, se utilizó el test t de Student. Se consideraron significativas estadísticamente la diferencias si el valor $p<0.05$. Los análisis fueron efectuados a través del software SPSS $v 17.0$ para Windows.

\section{RESULTADOS}

Concentración Total de Proteínas de Saliva no Estimulada

Los adultos tuvieron una concentración de proteínas en la saliva no estimulada de $3.2 \pm 1.7 \mu \mathrm{g} / \mu \mathrm{l}$, mientras que en los adultos mayores fue de $5.5 \pm 2.6 \mu \mathrm{g} / \mu \mathrm{l}$, diferencia que resultó estadísticamente significativa $(p<0.05)$ (Figura 1).

Al comparar los resultados según género, no se observaron diferencias entre hombres y mujeres en la concentración de proteínas, ni en los adultos, ni en los adultos mayores ( $p>0.05$ ) (Figura 1). Sin embargo, los hombres adultos mayores $(5.4 \pm 0.5 \mu \mathrm{g} / \mu \mathrm{l})$ tuvieron más altas cantidades de proteínas salivales que los hombres adultos $(3.0 \pm$ $0.4 \mu \mathrm{g} / \mu \mathrm{l})(\mathrm{p}>0.05)$ (Figura 1). Asimismo, las mujeres adultas mayores $(5.6 \pm 0.8 \mu \mathrm{g} / \mu \mathrm{l})$ mostraron mayor concentración de proteínas que las mujeres adultas $(3.8 \pm 0.4 \mu \mathrm{g} / \mu \mathrm{l})(\mathrm{p}<0.05)$ (Figura 1).

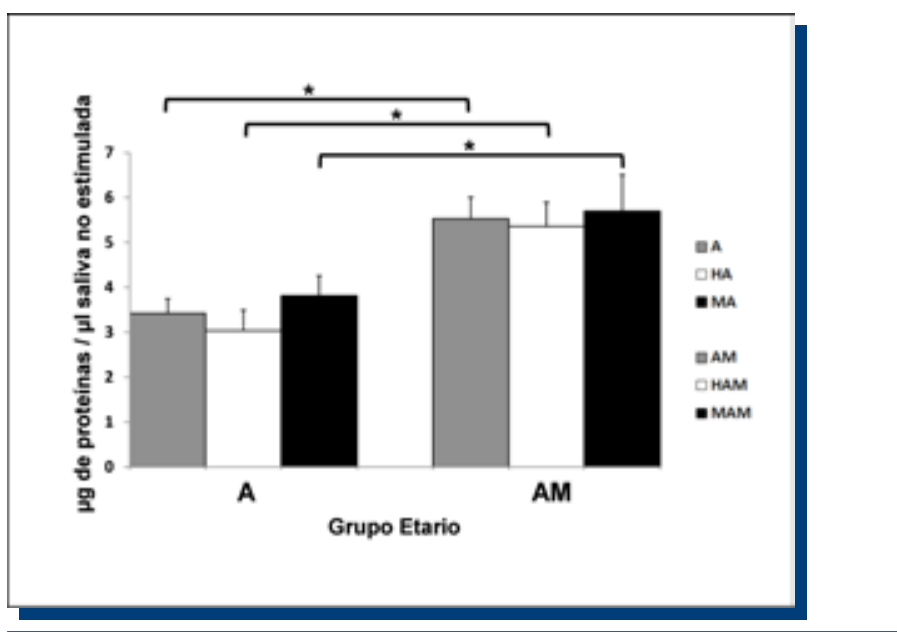

Figura 1. Concentración de proteínas en saliva no estimulada. Se determinó la concentración de proteínas a partir de muestras de flujo salival no estimulado de adultos $(\mathbf{A})$ y adultos mayores $(\mathbf{A M})$. La figura muestra las concentraciones proteicas ( $\mu \mathrm{g}$ proteína/ $\mu \mathrm{L}$ de saliva no estimulada) del total de A y AM (barras grises), hombres adultos (HA) y hombres adultos mayores (HAM) (barras blancas) y mujeres adultas (MA) y mujeres adultas mayores (MAM) (barras negras). Las barras representan el promedio \pm DS. Comparaciones entre los corchetes. ${ }^{*}=p<0.05$.

\section{Concentración Total de Proteínas de Saliva Estimulada}

Similar a lo observado para la saliva no estimulada, la concentración de proteínas salivales de los adultos fue menor $(p<0.05)$ que la de adultos mayores, con valores de $4.0 \pm 0.3 \mathrm{ug} / \mu \mathrm{l}$ y $4.9 \pm 0.4 \mathrm{ug} /$ $\mu \mathrm{l}$ respectivamente (Figura 2). Pese a que no se observaron diferencias significativas entre hombres y mujeres del mismo grupo etario $(p>0.05)$, los hombres adultos tuvieron menor concentración de proteínas $(p<0.05)$ que los hombres adultos mayores, con valores de $3.3 \pm 0.4 \mu \mathrm{g} / \mu \mathrm{l}$ y 4.4 $\pm 0.3 \mu \mathrm{g} / \mu \mathrm{l}$ respectivamente (Figura 2 ). Una similar tendencia se obtuvo entre mujeres adultas y adultas mayores, con $4.7 \pm 0.4 \mu \mathrm{g} / \mu \mathrm{l}$ y $5.5 \pm 0.5$ $\mu \mathrm{g} / \mu \mathrm{l}$, respectivamente $(\mathrm{p}<0.05)$ (Figura 2$)$.

\section{DISCUSIÓN}

Los resultados de esta investigación muestran que a medida que avanza la edad, aumenta el número de proteínas salivales totales, independientemente del sexo de las personas. Si bien, se asumió por mucho tiempo que la disminución en la cantidad de saliva es un proceso normal, propio del envejecimiento ${ }^{(23)}$, y esta alteración tendría un directo impacto sobre la composición de este fluido, hoy sabemos que en individuos sanos, el flujo salival se mantiene estable en relación al paso de 


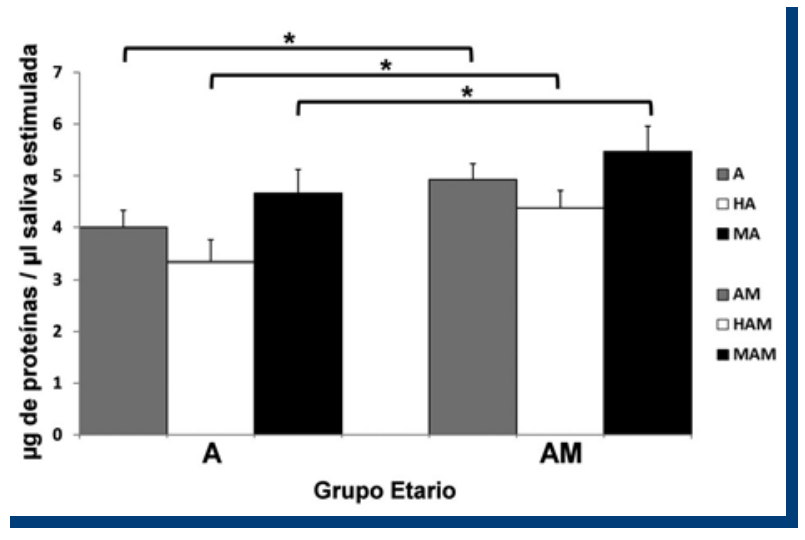

Figura 2. Concentración de proteínas en saliva estimulada. Se determinó la concentración de proteínas a partir de muestras de flujo salival estimulado de adultos (A) y adultos mayores (AM). Se muestran las concentraciones proteicas ( $\mu \mathrm{g}$ proteína/ $\mu \mathrm{L}$ de saliva estimulada) de A y AM (barras grises), hombres adultos (HA) y hombres adultos mayores (HAM) (barras blancas) y mujeres adultas (MA) y mujeres adultas mayores (MAM) (barras negras). Las barras representan el promedio \pm DS. Comparaciones entre los corchetes. ${ }^{*}=p<0.05$

los años ${ }^{(24)}$. Sin embargo, aún existe controversia respecto de la cantidad y variedad proteica salival en individuos de diferente edad y estado de salud general, ya que múltiples factores locales o sistémicos explican las variaciones en la composición química salival(25). A pesar de esto, diferentes autores concuerdan con que una disminución en el contenido proteico de la saliva representa un claro indicador de riesgo de caries $^{(9)}$.

La población de Chile y del mundo está experimentando cambios demográficos dramáticos ${ }^{(26)}$. Se espera un aumento importante en el número de adultos mayores para los años venideros, los que se esperan alcancen un $20 \%$ de la población para el año $2025^{(15)}$. De allí que el conocer las características del envejecimiento en la fisiología y la patología de la cavidad bucal resulte de alto interés. Dado que la saliva ha demostrado un rol protector frente a la caries y otras patologías orales ${ }^{(11)}$, variaciones en su composición pueden jugar un papel como factores de riesgo o actuar como agente etiológico. De hecho, los adultos mayores presentan significativamente más altas tasas de caries radiculares ${ }^{(27)}$ y un biofilm de composición diferente que individuos adultos ${ }^{(28)}$.

Dado que no existen estudios con resultados concluyentes sobre una cantidad o valor específico de referencia para el recuento de proteínas totales, además de ausencia de un método único estandarizado, la comparación de nuestros resultados con los obtenidos por otros autores es difícil.

En esa misma dirección, tampoco existe un protocolo para procesar las muestras salivales. En nuestro estudio, analizamos la concentración proteica tanto en muestras de saliva estimulada como no estimulada; lo cual resulta interesante si se piensa que ambos flujos tienen diferente origen glandular ${ }^{(29)}$, se secretan ante diferentes condiciones y presentan diferentes características bioquímicas y pese a ello, ambos muestran un comportamiento similar en cuanto a la concentración proteica según la edad.

Al contrastar los resultados según sexo, no es posible observar diferencias en las concentraciones proteicas. Con respecto al tema, en la literatura no existe evidencia sólida que muestre algún tipo de diferencia entre hombres y mujeres.

Diversas patologías sistémicas pueden afectar el flujo salival y consecuentemente la concentración de proteínas $^{(17-20,30)}$. A diferencias de otros estudios, los criterios empleados en el presente trabajo para la selección de pacientes, excluyeron aquellos que presentaran patologías sistémicas, para así evitar la influencia de esta en la concentración proteica y aislar mejor el efecto del proceso fisiológico del envejecimiento. Pese a lo anterior, futuros estudios deberían abordar la temática de las enfermedades sistémicas o del consumo de fármacos, de tal suerte de incorporar factores que son prevalentes para este grupo poblacional.

En relación al número de proteínas salivales específicas y envejecimiento, algunos autores sostienen que hay una disminución en los niveles salivales de Inmunoglobulina $\mathrm{M}$ e inmunoglobulina $\mathrm{G}$ asociadas a este proceso. Sin embargo, los valores para IgA se mantendrían estables conforme avanza la edad de los individuos ${ }^{(24)}$

El análisis de la concentración y luego del tipo de proteínas, junto a la obtención de un perfil proteico salival en relación a la edad, sería el primer paso para dilucidar un posible rol que tendría la presencia o ausencia de estas moléculas, en el proceso salud-enfermedad; permitiendo identificar proteínas salivales vinculadas a una enfermedad específica, como por ejemplo caries o enfermedad periodontal, ayudando a su diagnóstico, el monitoreo de su progresión, identificar grupos de riesgo susceptibles y planificar tratamientos.

\section{CONFLICTOS DE INTERÉS}

Los autores no declaran conflicto de interés.

\section{REFERENCIAS BIBLIOGRÁFICAS}

1. Llena-Puy $C$. The role of saliva in maintaining oral health and as an aid to diagnosis. Med Oral Patol Oral Cir Bucal, 2006; 11: E449-455.

2. Kaufman E. The diagnostic applications of saliva-a review. Crit Rev Oral Biol Med, 2002; 13(2): 197-212.

3. Denny $P$ et al. The proteomes of human parotid and submandibularsublingual gland salivas collected as the ductal secretions. J Proteome Res, 2008; 7: 1994-2006.

4. Yang LL et al. Comparative analysis of whole saliva proteomes for the screening of biomarkers for oral lichen planus. Inflamm Res, 2006; 55: 405-407.

5. Fleissig Y. Comparative proteomic analysis of human oral fluids according to gender and age. Oral Diseases, 2010; 16: 831-838.

6 . Rao PV et al. Proteomic identification of salivary biomarkers of type-2 diabetes. J Proteome Res, 2009; 8: 239-245.

7. Minarowski $Ł$ et al. Thiocyanate concentration in saliva of cystic fibrosis patients. Folia Histochem Cytobiol, 2008; 46: 245-246.

8. Hu S et al. Discovery of oral fluid biomarkers for human oral cancer by mass spectrometry. Cancer Genomics Proteomics, 2007; 4: 55-64.

9. Mungia $R$ et al. Interaction of age and specific saliva component output on caries. Aging Clin Exp Res, 2008; 20: 503-508.

10. Ambatipudi KS, Lu B, Hagen FK, Melvin JE, Yates JR. Quantitative analysis of age specific variation in the abundance of human female parotid salivary proteins. J Proteome Res, 2009; 8(11): 5093-5102.

11. Amerongen JGM, Bolscher \& ECI Veerman. Salivary proteins: Protective and diagnostic value in cariology? Caries Res, 2004; 38: 247-253.

12. Sivakumar T, Hand AR, Mednieks M. Secretory proteins in the saliva of children. J Oral Sci, 2009; 51(4): 573-580.

13. World Health Organization. Informe de la Segunda Asamblea Mundial sobre Envejecimiento: "Envejecimiento de la población: Hechos y cifras". Publicación de las Naciones Unidas ISBN 92-1-330176-6, Nueva York. 2002.

14. Servicio Nacional de Estadística. "Proyecciones y Estimaciones de Población. 1990 -2020. País y Regiones". Chile, 2008.

15. World Health Organization. Envejecimiento, derechos humanos y políticas públicas. ISBN 978-92-1-323215-6 LC/G2389-P, Santiago de Chile, 2009.

16. CELADE. Observatorio Demográfico CELADE-CEPAL. 2007.

17. González M, Montes de Oca L, Jiménez G. Cambios en la composición de la saliva de pacientes gestantes y no gestantes. Perinatol Reprod Hum, 2001; 15: 195-201.

18. Actis $A B$, Simbrón $A$, Brunotto $M$, Gómez de Ferraris ME. Concentración de proteínas totales en saliva de jóvenes consumidores sociales de alcohol. Acta Odontol Venez, 2006; 44(2): 171-175.

19. Gutiérrez Novoa P, Olivares Navarrete R, Leyva Huerta ER. Factor de crecimiento epidermal y proteínas totales en saliva de fumadores y no fumadores. Av Odontoestomatol, 2008; 24(6): 377-383.

20. Osorio González AY, Bascones Martínez A, Villarroel-Dorrego M. Salivary $\mathrm{pH}$ alterations in smoker patients with periodontal disease. $A v$ Periodon Implantol, 2009; 21(2): 71-75.

21. Banderas-Tabaray J, Gonzalez-Begne M, Sanchez-Garduño A, Millan-Cortez E, Lopez-Rodriguez A, Vilchis-Velazquez A. The flow and concentration of proteins in human whole saliva. Salud Pública Méx, 1997; 39(5).

22. Llena-Puy MC, Montaña-Llorens C, Forner-Navarro L. Condiciones óptimas de manipulación para la cuantificación de fibronectina en saliva. Med Oral, 2004; 9: 191-196. 
23. Vissink A, Spijkervet FK, Van Nieuw Amerongen A. Aging and saliva: A review of the literature. Spec Care Dentist, 1996; 16(3): 95-103.

24. Gati D, Vieira A. Elderly at greater risk for root caries: A look at the multifactorial risks with emphasis on genetics susceptibility. Int $J$ Dent, 2011; 68: 64-71.

25 . Schenkels L. Biochemical composition of human saliva in relation to other mucosal fluids. CROBM, 1995; 6: 161.

26. Pérez V, Sierra F. Biología del envejecimiento. Rev Méd Chile, 2009; 137: 296-302.

27. Sugihara N, Maki Y, Okawa Y, Hosaka M, Matsukubo T, Takaesu Y. Factors associated with root surface caries in elderly. Bull Tokyo Dent Coll, 2010; 51(1): 23-30.
28. Senpuku H, Sogame A, Inoshita E, Tsuha Y, Miyazaki H, Hanada N. Systemic diseases in association with microbial species in oral biofilm from elderly requiring care. Gerontology, 2003; 49(5): 301-309.

29. Lenander-Lumikari M, Loimaranta V. Saliva and dental caries. Adv Dent Res, 2000; 14: 40-47.

30. Sevón L, Laine M, Karjalainen S, Doroguiinskaia A, Helenius H, Kiss $\mathrm{E}$, Lehtonen-Veromaa M. Effec of age on flow-rate, protein and electrolyte composition of stimulated whole saliva in healthy, non-smoking women. Open Dent J, 2008; 2: 89-92. 